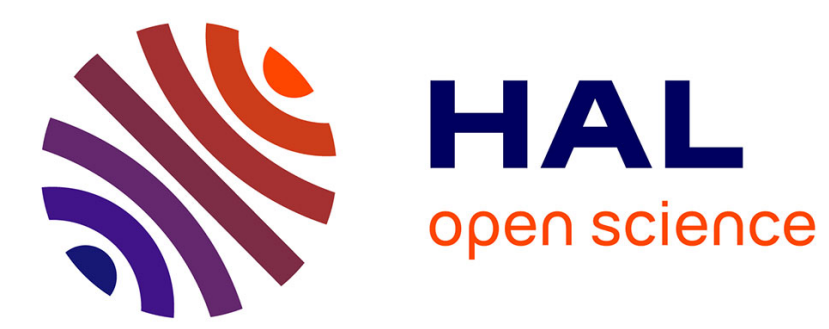

\title{
New insights on eye blindness and hand sight: Temporal constraints of visuo-motor networks
}

Yves Rossetti, Laure Pisella, Denis Pélisson

\section{To cite this version:}

Yves Rossetti, Laure Pisella, Denis Pélisson. New insights on eye blindness and hand sight: Temporal constraints of visuo-motor networks. Visual Cognition, 2000. hal-02152312

\section{HAL Id: hal-02152312 \\ https://hal.science/hal-02152312}

Submitted on 11 Jun 2019

HAL is a multi-disciplinary open access archive for the deposit and dissemination of scientific research documents, whether they are published or not. The documents may come from teaching and research institutions in France or abroad, or from public or private research centers.
L'archive ouverte pluridisciplinaire HAL, est destinée au dépôt et à la diffusion de documents scientifiques de niveau recherche, publiés ou non, émanant des établissements d'enseignement et de recherche français ou étrangers, des laboratoires publics ou privés. 
VISUAL COGNITION, 2000, 7 (6), 785-808

\title{
New insights on eye blindness and hand sight: Temporal constraints of visuo-motor networks
}

\author{
Yves Rossetti, Laure Pisella, and Denis Pélisson \\ Espace et Action, INSERM, Bron, France
}

\begin{abstract}
Pioneer experiments on saccadic suppression have shown that this effect is not followed by motor disorientation: Conscious perception of a target displacement can be dissociated from correct manual target reaching. It has subsequently been demonstrated that movement corrections with the same latency and spatial characteristics can be produced in conditions allowing perceptual awareness of perturbation of a target as in condition inducing saccadic suppression. In addition to the qualitative dissociation between motor performance and conscious awareness, quantitative temporal dissociations in action can be observed by manipulating different features of the visual target. When the target of an ongoing simple action is perturbed, a temporal advantage is found for responses to perturbations of location relative to colour and shape. Furthermore, there seems to be a temporal advantage for automatic motor corrections made in response to a target displacement as compared to other responses (other ongoing movement adjustments, movement interruption, conditional motor response such as pressing a key, verbal response, delayed matching-to-sample tasks). Thus, this paper reviews evidence for the fact that the temporal characteristics of any given response to a stimulus are dependent both on the sensory processes and on the type of response generated. Accordingly, identification responses (such as verbal report) to a visual stimulus are much slower than motor corrections of an ongoing movement in response to a target location change because of different processing times of the stimulus features ("What" compared to "Where") and of the response itself ("What" compared to "How"). The existence of two continua (What/Where and What/How) is proposed between these two extreme stimulusresponse combinations. This model may be a useful framework to better understand visuo-motor transformations and the network of connections between visual and motor areas.
\end{abstract}

Please address all correspondence to Y. Rossetti, Institut National de la Santé Et de la Recherche Médicale, Unité 534: Espace et Action, 16 avenue Lépine, Case 13, 69676 Bron, France. Email: rossetti@lyon151.inserm.fr

The authors wish to deeply thank Ken Knoblauch (Lyon) and Rob MacIntosh (St Andrews) for their thoughtful comments on an earlier version of this text, and Jean-Louis Borach for his help with illustrations. This work was supported by INSERM, Région Rhône-Alpes (Programme Thématique, prioritaire 97-12-591), CNRS (GIS Cognisciences), and COGNITIQUE. 


\section{DISSOCIATION BETWEEN CONSCIOUSNESS AND MOVEMENT CORRECTION}

The performance of simple visually guided acts such as reaching to an object with the hand relies on extensive processing of information about object location, motion, orientation, shape, and texture. This complex visual analysis is at odds with the facility of normal human beings to plan and execute such goaldirected actions, without even being fully aware of their own motor acts. In fact, a great deal of underlying visuo-motor mechanisms can function automatically and do not require consciously generated decisions from the subject. In this paper, we review some findings that illustrate a dissociability between the neural processes that participate in generating a conscious percept of the visual environment and those that automatically initiate and steer motor responses within this environment.

The automaticity of goal-directed motor acts is particularly evident for saccadic eye movements since most of them are not consciously elicited. This is already true for low amplitude saccades (such as the microsaccades occurring during fixation). As shown later larger saccades performed in response to a target jump also exhibit an automatic component. Indeed, the orientation of gaze toward an eccentric target (presented as a step from an initial fixation point) is achieved by a primary saccade that usually undershoots the target (Henson, 1978), followed by another saccade that corrects for the residual error. Interestingly, the latency of these corrective saccades is about half that of primary saccades (Becker \& Fuchs, 1969). One possible explanation of this latency difference is that the direction and approximate amplitude of corrective saccades are predictable and can be programmed before the actual completion of the primary saccade. However, it has been shown that when the target unexpectedly jumps again during the primary saccade (a so-called "double-step" stimulus) to render the post-saccadic error unpredictable, the corrective saccade is elicited as fast as normally (Prablanc \& Jeannerod, 1975). Only when the second jump is larger than about 4 deg is the latency of the corrective saccade as large as that of initial saccades. Prablanc and Jeannerod suggested that the planning of a corrective saccade at the end of the primary saccade relies on an unconsciously detected signal of the remaining error, which can by-pass the normal decision process (provided this error does not exceed values related to the tolerated primary saccade inaccuracy). Furthermore, subjects cannot consciously process information about any perturbation of the target position that occurs during the primary saccade because of the saccadic suppression phenomenon described later. As early as 1900, Dodge noted that, if the head remains stationary, seeing one's own eye motion in a mirror is impossible. Indeed, one can easily see one's own eyes in successive positions, but never in motion. Later, psychophysical studies revealed that human subjects are unaware of displacements occurring within the visual world if these 
displacements occur during the saccade (e.g., Bridgeman, Hendry, \& Stark, 1975). Saccadic suppression thus refers to the decrease of perceptual capabilities during saccades (Campbell \& Wurtz, 1978). Saccadic suppression has been widely cited as one of the central mechanisms maintaining the stability of the visual world despite saccadic eye movements (Bridgeman, Van der Heijden, \& Velichkovsky, 1994). Experimental paradigms that take advantage of saccadic suppression by using double-step stimuli, in which the second step is synchronized with the first saccade, have permitted investigation of automatic mechanisms regulating goal-directed motor responses.

As with saccades, reaching movements of the arm have been described in terms of two phases (see Desmurget, Pélisson, Rossetti, \& Prablanc, 1998). The initial phase of reaching is under feedforward control, and the remaining phase is subjected to several sensory feedback loops. The role of these feedback loops has been investigated by abruptly changing the location of the stimulus in conditions that either do or do not allow the conscious detection of the target perturbation by the subject. In one early experiment, subjects were asked to point to the position of a target that had been displaced during the saccade (by a mirror induced motion) and then extinguished prior to onset of the arm movement (Bridgeman, Lewis, Heit, \& Nagle, 1979). The same kind of dissociation between perceptual and motor output was found as for eye movements: pointing movements following a target jump remained accurate irrespective of whether this displacement could be verbally reported or not. These experiments therefore suggested that two psychophysically separable visual systems can be distinguished, one for a "cognitive" response, and another one for immediate motor behaviour (Bridgeman, 1991, 2000; Bridgeman, Peery, \& Anand, 1997; Desmurget et al., 1998; Rossetti, 1998; Pisella \& Rossetti, 2000).

Experiments aimed at elaborating the type of sensory information involved in motor control have further explored this last phenomenon (Goodale, Pélisson, \& Prablanc, 1986; Pélisson, Prablanc, Goodale, \& Jeannerod, 1986) (Figure 1). In these experiments, the authors asked subjects to point as fast and as accurately as possible to visual targets presented in the dark. On half of the trials, the target simply jumped from a central position to a randomly selected position in the peripheral visual field. On the other trials, the target made a second jump, time-locked to the orienting saccade that slightly led onset of the hand movement. This second step was parallel to the first and was directed either in the same or the opposite direction, requiring respectively an increase or a decrease in pointing amplitude. Subjects were never aware of the second target jump, and could not even guess its direction when tested with forcedchoice verbal responses. Nevertheless, it was clearly shown that not only the eye (after a corrective saccade) but also the hand reached the target in all cases, although both the eye and the hand had initially been prepared to aim at the first target. Strikingly, subjects were never aware of performing motor corrections. Similar results were obtained when the second target jump altered the direction, 
A
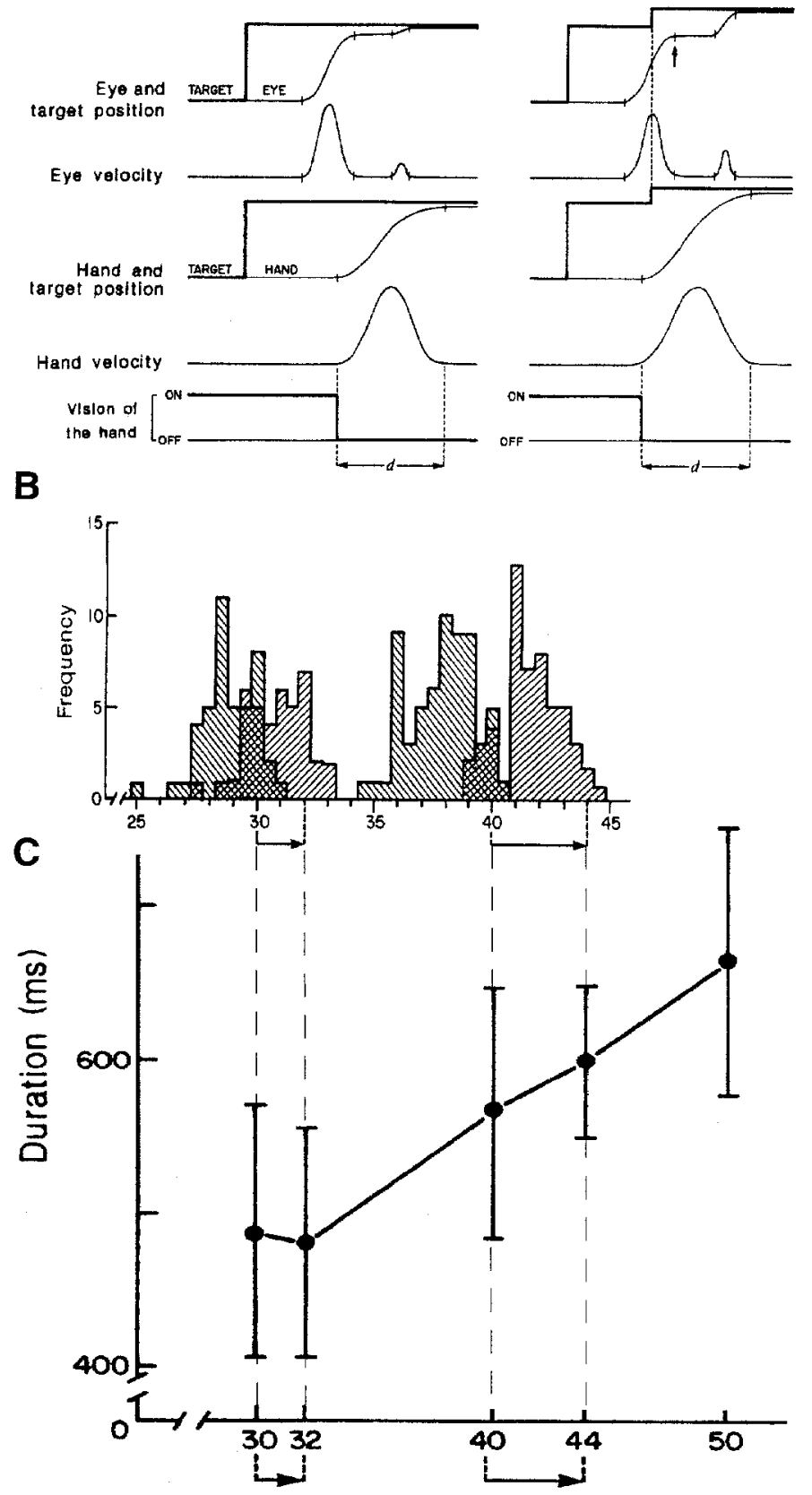

Amplitude $(\mathrm{cm})$ 
rather than the amplitude, of the required movement (Prablanc \& Martin, 1992). Again, subjects were unable to show a conscious detection of the visual change induced by the perturbation in target location nor did they show detection based on the correction of their own motor response. It was concluded that vision of the moving hand was not necessary to control the ongoing movement and, more strikingly, that movement trajectory could be updated without the subject becoming aware of the correction. These studies thus demonstrated that perception of target position could be dissociated from visuo-motor responses directed to that target, that is, that different types of visual computation are made for visual perception and for visuo-motor control (see reviews in Desmurget et al., 1998; Goodale \& Haffenden, 1998; Rossetti, 1998, 2000; Pisella \& Rossetti, 2000).

The experiments reviewed in this section suggest that certain types of sensory information can be used implicitly during goal-directed movements. Accordingly, it may be hypothesized that two different systems underlie conscious perception of target perturbations and the use of visual information about the perturbation to effect a change in the motor response. We refer to the latter as hand sight.

\section{TEMPORAL DISSOCIATIONS BETWEEN CONSCIOUSNESS AND HAND SIGHT}

Some of the work supporting the dissociation between movement production and "higher level" processing of the target has emphasized the influence of temporal parameters on performance (for reviews see Bridgeman, 2000; Rossetti, 1998, 2000; Pisella \& Rossetti, 2000). Let us consider here the time differences between these two types of sensori-motor process that have been

Figure 1 (Opposite). Saccadic suppression and hand pointing performance. A: Experimental procedure: Schematic representation of single- and double-step trials randomly presented during an experimental session. In all trials, the target was displaced from a central position to a randomly selected position in the right hemi-field and vision of the hand was turned off at the onset of the hand response. In double-step trials, the peripheral target jumped again to the right at the time the saccadic eye response reached its peak velocity, i.e., nearly at hand movement onset. The second target step represented $10 \%$ of the first step amplitude and was not detected consciously by subjects. B: Distribution of hand pointings. Distributions of the endpoints of hand pointing responses, pooled over four subjects, towards single-step targets at 30 and $40 \mathrm{~cm}$ and towards double-step targets (30-32 and 40-44 cm). Note that pointings to double-step targets undershoot the final target location (a characteristic of hand movements performed without visual feedback) to the same extent as pointings to single-step targets, demonstrating the existence of corrective processes compensating for the target perturbation. C: Duration of hand pointings. Relationship between hand pointing duration (mean and standard deviation) and target step amplitude for three single-step targets $(30,40$ and $50 \mathrm{~cm})$ and for two double-step targets $(30-32$ and 40-44 cm). Same responses as in B. It can be seen that the same relationship accounts for both types of trials, indicating that motor correction in response to a target perturbation (see panel B) is not related to an increased pointing duration (Modified from Goodale et al., 1986 and Pélisson et al., 1986). 
reported in several psychophysical experiments (related neuropsychological data will be presented further later).

A first kind of difference is related to the duration of memory traces that follow visual inputs. A difference in memory trace duration for the two types of sensori-motor process has been suggested in several studies that have introduced a delay between the target presentation and the motor response. As an illustration, we will first consider data by Bridgeman (1991). Ten subjects were presented with a visual stimulus consisting of a spot enclosed within a rectangular frame. The target spot was randomly presented at one of five positions relative to the observer; the rectangular frame could be located straight ahead with respect to the observer, or located asymmetrically to the left or right of the straight ahead direction. Both target and frame were simultaneously presented for one second, after which subjects were asked either (1) to judge which target had been presented (perceptual response) or (2) to aim at the target with a pointer (motor response). Perceptual and motor responses were recorded in different trials that were randomly mixed within experimental sessions. These responses were made immediately after the removal of the visual stimulus or $4 \mathrm{~s}$ after its offset, in two separate experiments. The results were different for perceptual and motor responses. Perceptual responses were systematically affected by the position of the frame such that the target was perceived as being closer to the nearest border of the frame, relative to its absolute position (Roelofs effect). This effect was observed for all subjects in the immediate response experiment and for almost all (eight out of nine) subjects in the delayed response experiment. In contrast, motor responses were differentially affected depending upon their delay from target presentation. Responses delayed by $4 \mathrm{~s}$ were, in eight out of nine subjects, affected by the illusion induced by the position of the visual frame, suggesting that target location was influenced by allocentric cues. In the case of immediate responses, five subjects relied on egocentric cues for locating the target such that their hand movements were unaffected by frame position, whereas the remaining five subjects had biased pointing responses, which demonstrated an influence of allocentric information on target localization. These results clearly indicate that the processing of visual inputs for motor control can depend upon the delay of the motor response, with an increased propensity to use allocentric cues when this delay increases. This increased propensity suggests that delayed motor responses rely on a visual representation resembling the one leading to perceptual responses.

More recently, it has been shown that delayed pointing movements towards a visual or a proprioceptive target are influenced by the geometrical configuration of the target array used during the experimental session (review in Rossetti, 1998). Specifically, the spatial distribution of the endpoints of delayed-pointing responses to a given target, as measured by the main orientation of the confidence ellipse fitted to the scatter, varies according to the context (location of 
the other targets) (see Figure 2). Indeed, the confidence ellipses for movements directed toward the same target but recorded in two separate sessions (with different contexts) can lie orthogonal to each other when only the orientation of the target array differs between these sessions (Rossetti \& Régnier, 1995; Rossetti, Gaunet, \& Thinus-Blanc, 1996; Rossetti \& Procyk, 1997). In contrast, no such contextual influence is observed on endpoint distribution for immediate pointing movements. This strongly suggests that delayed action is more likely to rely on location information based on an allocentric representation of space, even if no external frame of reference is explicitly provided to the subject.

A second type of temporal difference that distinguishes visual processing for movement production from "higher level" visual processing is related to the timing of recruitment. One interesting feature of the automatic visuo-motor processing is the particularly short latency that is measured between a target jump occurring in the environment and the motor reaction to it. Visuo-motor delays have been estimated at about $110 \mathrm{~ms}$ by detailed kinematic analyses (Prablanc \& Martin, 1992). This value is strikingly similar to that obtained when the target jump is not synchronized with the saccade and can therefore be consciously detected by the subjects, both for pointing movements (Komilis, Pélisson, \& Prablanc, 1993), and for reach-to-grasp movements (perturbation of either object location in Paulignan, MacKenzie, Marteniuk, \& Jeannerod, 1991 or object orientation in Desmurget et al., 1996). Similar experiments using a pseudo-change in object size synchronized with movement onset show a comparable latency for the motor reaction of the arm transport component (note, however, that latencies of about $330 \mathrm{~ms}$ were observed for the grasp component, indicating that changes in grasp with respect to intrinsic object properties were processed slower than a change in location) (Paulignan \& Jeannerod, 1991). These results indicate that the short latencies of reaching and grasping corrections are not dependent on whether target perturbation is consciously perceived or not. The processes underlying motor corrections and conscious perception may therefore operate in parallel.

The high speed of motor corrections has also been stressed by studies that have attempted to relate motor correction time to the delay in subjective awareness of the perturbations (Castiello, Paulignan, \& Jeannerod, 1991; Castiello \& Jeannerod, 1991). In these experiments, subjects reached to and grasped an object that could be perturbed in location or size at the start of the hand transport component. Subjects were instructed both to correct their motor responses and to produce a simple vocal utterance ("Tah!") as soon as they could detect the perturbation. Hand motor reaction time and vocal reaction time were compared. As in the initial experiments of Paulignan and Jeannerod (1991) and Paulignan et al. (1991), onset of the correction of the hand transport component lagged object displacement by about $110 \mathrm{~ms}$, and this delay increased to about $280 \mathrm{~ms}$ for the adjustment of the grasp component to a change in object size. 

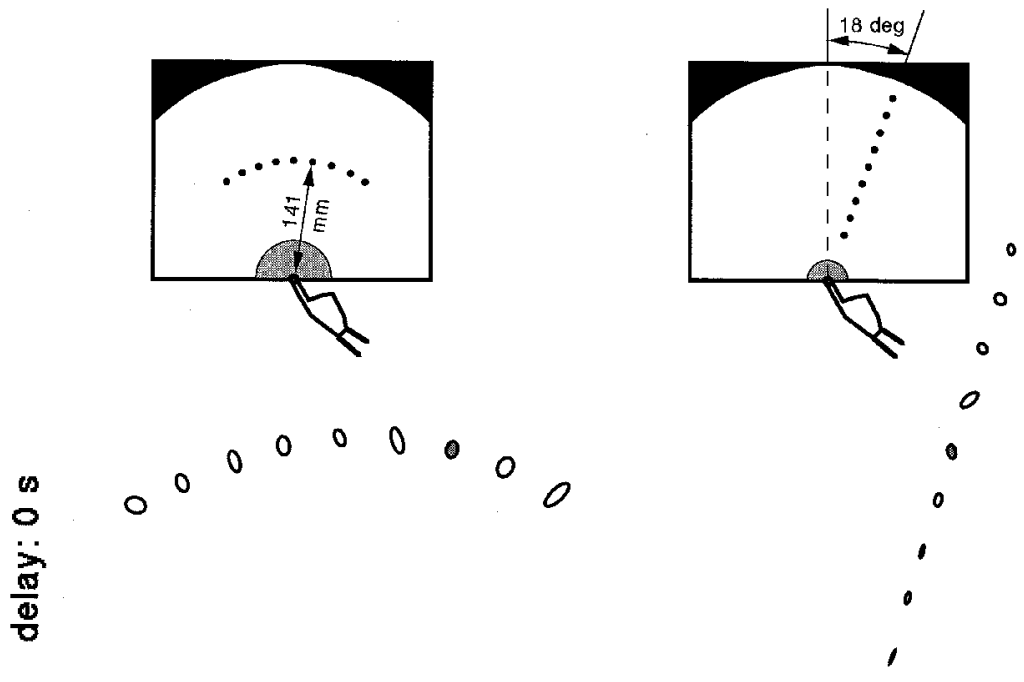

0

$+$

Starting point

Starting point

$0 \mathrm{~cm}$

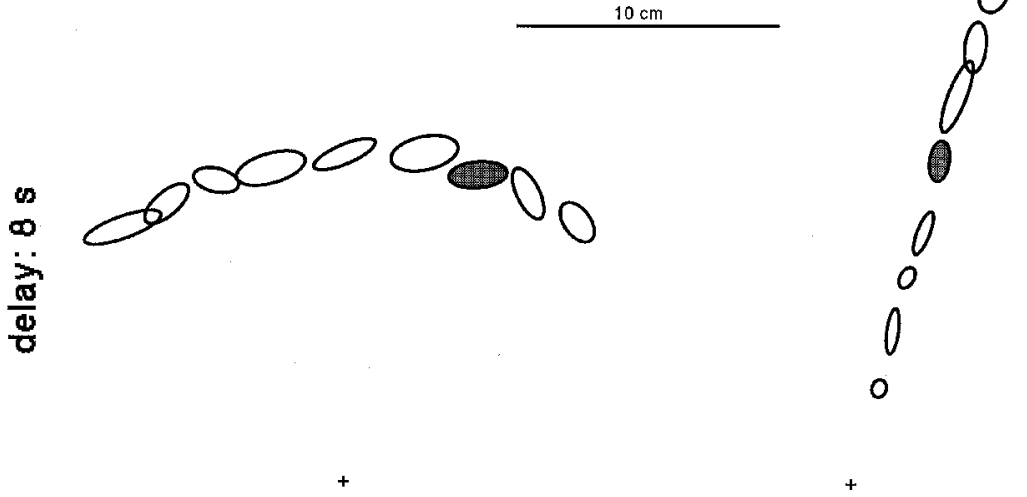

Figure 2. Typical results obtained for immediate $(0 \mathrm{~s})$ vs. delayed $(8 \mathrm{~s})$ pointing to briefly flashed visual stimuli. In these experiments two different target arrays (arc and line) were used in separate sessions and the various delays were presented in a randomized order within one session. In addition to the expected increase in endpoint variability observed after the delay, a clear interaction is observed between the effect of the target array used in a given session and the effect of the delay. For immediate pointing, the ellipse main axis depicting pointing scatter tends to be aligned with the finger starting position for both target arrays (i.e., aligned with movement direction). But for delayed pointing, the ellipse main axis became orthogonal to movement direction for the arc array whereas it remained aligned with movement direction for the line array. This result indicates that the scatter of delayed pointing was highly dependent on the shape of the target array, suggesting that the accuracy of delayed pointing towards a given target is altered by information integrated over trials about the other target locations (from Rossetti, 1998). 
However, the vocal responses occurred in both cases about $420 \mathrm{~ms}$ after the object's perturbation. The authors concluded that the motor response to a stimulus change can be dissociated in time from its conscious detection with the latter lagging far behind the former. However, the differences in reaction time observed between these two responses may not only depend on the distinction between automatic and conscious processing, but also on the difference in the motor responses themselves, since the motor pattern generation required by online reach-to-grasp corrections and vocal responses may not follow identical time courses. This issue of the time constraints attached to different motor outputs will be further addressed later.

We have shown in this section that the qualitative distinction raised earlier between motor reactions and conscious reports can be extended to temporal dissociations between the different responses, when both sensori-motor and perceptual systems have access to the relevant visual information. Both the exploration of delayed actions and the temporal analysis of movement kinematics suggest that (1) direct sensori-motor responses can be recruited faster than conscious perceptual reports and that (2) motor representations have shorter life times (see also Rossetti, 1998, 2000; Pisella \& Rossetti, 2000).

\section{NEUROANATOMICAL CORRELATES OF HAND SIGHT}

The previously discussed dissociations between visual awareness and fast motor corrections further suggest that the underlying neural pathways are distinct and characterized by different processing speeds. The hypothesis of two parallel visual pathways operating at different speeds is supported by a wealth of data dealing with the "ventral/dorsal" distinction, that we will review here.

There is well established converging evidence from various experimental areas for a functional dissociation of visual processing between a "ventral" and a "dorsal" stream. Beyond the occipital areas, extensive visual processing is mainly divided between temporal and parietal areas, and the anatomical connectivity of visual areas in the cortex of the monkey is consistent with parallel processing of visual inputs (in order to see colour Plate 1A, please click here). Two main functional interpretations for this anatomical segregation have been proposed (see reviews in Milner \& Goodale, 1995; Jeannerod \& Rossetti, 1993; Milner \& Goodale, 1993; Pisella, Arzi, \& Rossetti, 1998; Ungerleider \& Mishkin, 1982). On the one hand, the anatomical segregation has been considered to correspond to the sensory processing of intrinsic vs. extrinsic stimulus attributes (object vs. spatial vision, or What/Where), on the basis of lesion experiments performed in the monkey. On the other, it has been interpreted as reflecting the goals of visual processing (identification vs. action, or What/ How), on the basis of the neuropsychological double-dissociation between 
visual agnosia and optic ataxia (respectively impairing specifically the identification of objects or the visuo-motor behaviour directed to them). We will later see that these two views may not be fully incompatible.

In addition to anatomical and functional distinctions, recent single unit studies in the monkey also provide evidence for a temporal dissociation between two visual pathways. Comparison of visual response latencies at various locations in the monkey visual system have led Nowak and Bullier (1997) to distinguish two groups of visual areas. Parietal areas from the dorsal stream, projecting onto the pre-motor cortex, exhibit particularly short visual latencies (about $40-80 \mathrm{~ms}$ ) as compared to other pre-striate areas. In their extensive review of the literature, the dorsal pathway projections towards frontal areas is therefore referred to as the "Fast Brain", whereas temporal areas are described as the "Slow Brain" (about 100-50 ms). As stressed by Nowak and Bullier (1997), the visual latencies do not match a hierarchical model of purely anatomical organization, but rather follow the distribution of magnocellular and parvocellular inputs. The speed of the occipito-parietal processing seems to be explained by the faster conductivity of the magnocellular channel almost exclusively activating this dorsal stream and by the numerous by-pass connections existing in it, whereas the ventral stream seems to be connected in a more serial fashion (Nowak \& Bullier, 1997; Schall et al., 1995). Neuroanatomical tracing has indeed shown that parietal areas of the dorsal stream project directly to the dorsal premotor cortex whereas the ventral stream projects only indirectly to ventral premotor cortex via the ventral prefrontal cortex (Schmolensky et al., 1998; Schwartz, 1994; Tanné et al., 1995).

With respect to the temporal issues raised here, it is interesting to note that the effect of human brain lesions on visuo-motor skills (How) can depend upon the delay of the response. The residual visuo-motor performance reported following an impairment of the ventral stream is no longer observed when the response is performed after a delay (in cases of visual agnosia, (Goodale, Jakobson, \& Keillor, 1994) and blindsight; Perenin \& Rossetti, 1996; for a review see Rossetti, 1998). The results of these neuropsychological explorations suggest that the dorsal stream gives rise only to short-lived representations, which may be superseded, in delayed movements, by other representations building up. The representations built in the ventral stream (via ventral projections onto premotor areas) may then override these motor representations for delayed movements.

Psychophysical investigations have confirmed a "What/Where" temporal distinction. Tanaka and Shimojo (1995) have shown that choice reaction times for location discriminations are shorter than those for colour discriminations, which suggests that the location system (extrinsic visual attribute processing) is faster than the colour system (intrinsic visual attribute processing). An advantage for the location system has also been reported with change detection tasks: During the visual exploration of a series of simple scenes, the detection of a 
change in object identity (concerning a single object or a switch of position between two objects) is rather poor. By contrast, a change in location of a single object is much more readily detected (Simons, 1996). Simons suggested that, as compared to visual memory of object properties (related to semantic processing), visual memory for object location does not depend on an effortful abstraction. Although an inter-stimulus interval was used, which strongly departs from the saccadic suppression paradigm and other movement control studies, this result is compatible with the idea that change in location is processed more efficiently than changes in intrinsic stimulus attributes.

The work reviewed here further supports the idea that two main visual pathways can be described within the brain. These two systems can be linked to distinct cortical areas and they exhibit different latencies of response to visual stimuli. It should be noticed, however, that beyond the well-known dichotomy between the so-called "dorsal" and "ventral" streams there are numerous interconnections between these two pathways. In addition, the respective contributions of magnocellular and parvocellular inputs to these two systems are not of an all-or-none character. These observations will lead us to suggest that gradients of sensory and motor processing would better reflect the true complexity of visual processing.

\section{TESTING THE SENSORY AND MOTOR COMPONENTS TO HAND SIGHT}

We have already considered the possibility that differences in the timing of visual tasks may depend partly on the nature of the response made to the stimulus. We will now examine the effect of object properties on response latencies in the motor context. A recent experiment was designed to check the ability of the motor system to react to typical dorsal and ventral visual attributes: namely location and colour. The manipulation of these features was aimed at comparing how dorsal and ventral features are integrated in action and at investigating the timing of their processing. Three types of perturbations were applied to an initial green target to which a pointing response was made. The target could change colour, location, or both, and subjects were instructed to touch the green targets and not the red ones. Consequently, (1) when the target was displaced, subjects had to correct their movement to the new location, (2) when the target turned to red, subjects had to stop their ongoing pointing, (3) when the target was perturbed simultaneously in both location and colour, subjects again had to interrupt their movement in order not to touch the red target (Pisella \& Rossetti, 2000). Because of the expected faster processing of location as compared to colour, it can be predicted that the double-perturbation will create a conflict between the fast correction response to the location perturbation, and a slower stop response to the colour change. In each condition, trials with and without perturbation were randomly presented with relative probabilities of $20 \% / 80 \%$. 
Subjects were requested to complete their response within an instructed temporal window (maximal movement time of 200, 250, 300, 350, and $400 \mathrm{~ms}$ were tested in five separate sessions), and a feedback tone signalled when the movement was too slow. Analysis of the performance of six subjects showed that, for pointing responses to the double perturbation with movement times ranging from 200 to about $280 \mathrm{~ms}$, subjects actually pointed to the displaced target that was formerly green and then turned to red, and that they reached this target as frequently as in response to simple location perturbations. Only slower movements were interrupted. This indicated that on-line motor corrections are so automatic that they may be executed even when subjects are explicitly instructed to block their motor act in response to a perturbation. However, because the instructions given to the subjects delineated two stimulus/response combinations (location/correction and colour/interruption), the fact that automatic motor corrections were initiated more quickly than the movement interruption could be related to the different stimulus attributes (location vs. colour change) and/or to the requested responses (correction vs. interruption). Therefore, applying the same methods as previously (pointing tasks divided into five separate sessions with pre-instructed movement times), we next tracked the temporal differences between responses made in several conditions, combining the two types of stimulus perturbation (change of target colour or location) and the two types of response instruction (correction or interruption of the ongoing movement). In a colour-stop condition, one group of subjects was instructed to interrupt their pointing movement in response to a change in target colour. In a location-stop condition, interruption was requested in response to a change in target location. In a location-go condition, a third group of subjects was instructed to redirect their movement to the secondary target location (see Figure 3A). The number of touches to the initial location of the target (errors) in perturbed trials was computed separately for each condition and each session. As expected, the percentage of errors for all conditions was close to $100 \%$ in the fastest session (mean observed movement time about $175 \mathrm{~ms}$ ). The main results from the comparison between conditions can be summarized as follows. First, the relationships between the absence of reaction to the perturbation on the one hand, and movement time (mean sessions) on the other hand, were very similar in the two location conditions, even though the instructions regarding the motor response ("go" or "stop") were obviously different. Second, when compared to the location conditions, the colour condition revealed that reactions to the perturbation appeared only at a longer movement time (Figure 3B). As seen from the horizontal shift between the curves, this time lag for the colour condition was observed over the whole range of movement times. These results suggest that the performance obtained in a given session was primarily affected by the stimulus being manipulated, whereas the timing of the motor reactions was not consistently affected by the instruction. A stimulus effect was thus clearly demonstrated between colour and location based processes, with a temporal lag of 


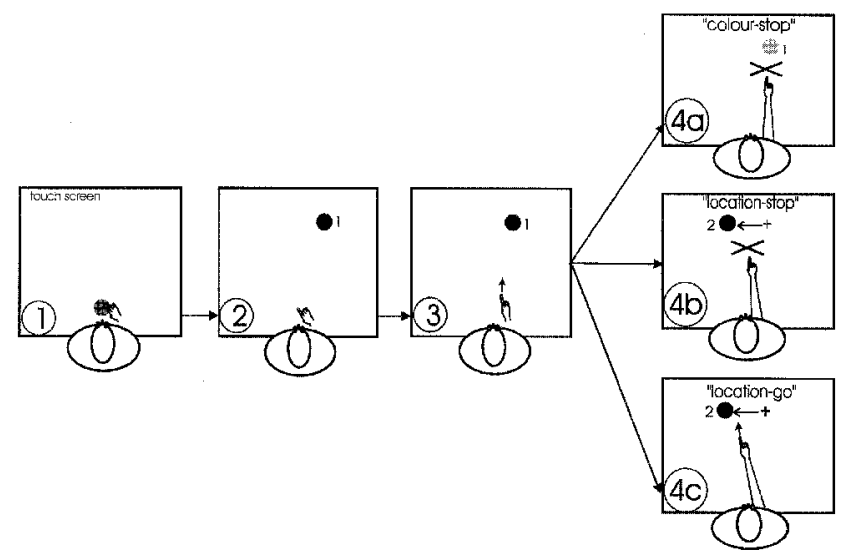

B

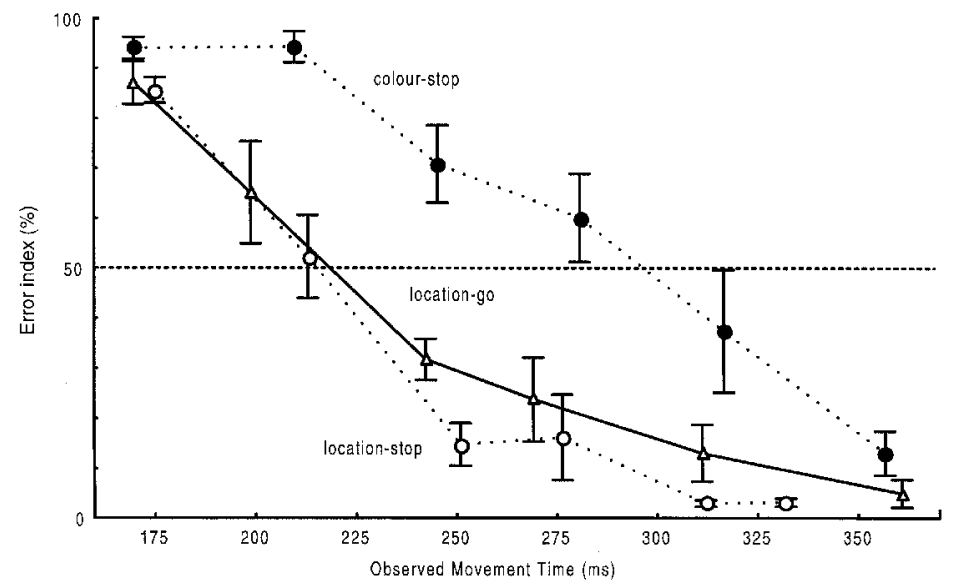

Figure 3. The temporal advantage of location during action. A: Experimental procedure. The first three steps of each trial are common to all conditions, i.e., up to finger movement onset. During step number 4 movement onset could trigger a change in target location ( $4 b$ and $4 c)$ or in target colour (4a), with a probability of $20 \%$. In the colour-stop condition (4a), subjects were instructed to stop their pointing in response to the target change in colour. In the location stop condition (4b), subjects were instructed to stop in response to the target change in location. In the location-go condition (4c), subjects were instructed to correct their pointing in response to the target change in location. The comparison between location-stop and colour-stop provides an estimate of the stimulus effect. By comparing locationstop and location-go, the effect of the instruction was evaluated. B: The timing of the first reactions observed in each session. The number of touches made to target 1 (error index) is plotted against the observed mean movement time for each session and for each condition (intersubject mean and standard error). One may anticipate errors to be close to $100 \%$ in the fastest sessions (100\% touch to initial target), and close to $0 \%$ in the slowest sessions (i.e., about $100 \%$ pointings redirected to the new target location or interrupted, according respectively to the "go" or to the "stop" instruction). This graph illustrates the differential evolution of errors across sessions between the colour condition and both location conditions. Departure from $100 \%$ occurs later for the colour condition. In the same way the $50 \%$ value is reached more than $50 \mathrm{~ms}$ earlier in the two location perturbed conditions. (Modified from Pisella et al., 1998.) 
about $50 \mathrm{~ms}$ for the former. Similar experiments have provided evidence that a perturbation of target shape is processed more slowly than a location change (Pisella, Kozawa, Susami, \& Rossetti, 2000). We have already presented above some evidence for an advantage for location discrimination relative to the discrimination of other stimulus attributes in a target detection task (Simons, 1996; Tanaka \& Shimojo, 1995). These experiments have shown that a similar stimulus effect can be observed in the context of a goal-directed action paradigm (Pisella et al., 1998; Pisella \& Rossetti, 1997). Further analyses of data obtained in our simple perturbation experiments have shown that the similar time course of the error rate observed for the location-go and the location-stop conditions can be explained by the unexpected occurrence of automatic corrections (performed in a precise temporal windows and against the interruption instruction) in the location-stop condition (Pisella et al., in press). A specific analysis of these automatic corrections revealed that the difference observed between the location-go and the colour-stop conditions could be decomposed into a $50 \mathrm{~ms}$ lag between the location and the colour-based processes and a 30 ms lag between the go and the stop responses (Rossetti \& Pisella, 1998). This preliminary result strongly supports the conclusion that the difference in timing reported in many studies reviewed here can be explained by a contribution of the timing of both stimulus and response processes.

\section{DISCUSSION}

Taken together, the data summarized here indicate that there are two main differences between on-line motor corrections and other responses. The first difference between these two types of behaviour is qualitative, in that the motor system seems to benefit from a favoured, and sometimes exclusive, access to visual information (as shown by the occurrence of unconscious corrections). In addition, interesting quantitative differences can be revealed by temporal analyses, motor corrections to location being the fastest response observed in all the experiments reviewed here. On the other hand, the last results presented here have also shown that target location is processed at a very privileged speed by the visual system.

The temporal analysis of various stimulus and response processes suggests that we may need to go beyond the simple distinction between two processing systems (What and Where, or What and How). At present, the available data would indeed favour the idea that the processing time associated with a specific task (defined by a stimulus-response combination) depends on the location of the stimulus attribute and the response type within a continuum rather than between dissociated systems. The experimental evidence reviewed here revealed a first continuum concerned with the stimulus processing time. Along this continuum, the "dorsal" attributes are processed faster than "ventral" ones. This gradient seems to be valid for a series of tasks ranging from online motor 
corrections to change detection. A second continuum appears to exist for response processing: Action correction seems to benefit from a faster processing than conditional motor responses and verbal descriptions. These latter responses are rooted in higher cognitive levels requiring a conscious perception and a symbolic analysis of the visual information, hence requiring additional time consumption.

The addition of the delays inherent to these two factors provides a global processing time for a given task. The location-correction combination can be conceived as one extremity of a set of possible combinations (or visuo-motor tasks) arranged in a gradient of time (see Figure 4). Thus the combination of the

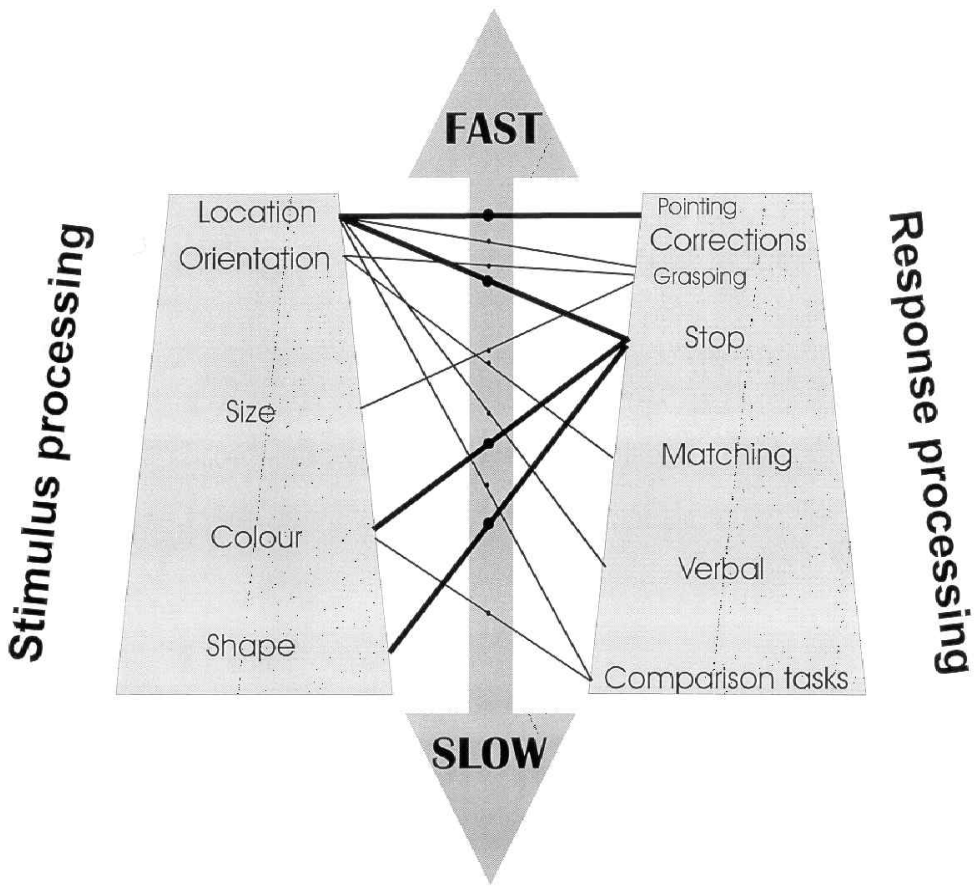

Figure 4. How two temporal gradients are integrated into the visuo-motor response. This figure attempts to sketch the temporal relationship of various visual attribute processing, the temporal relationship of various response generation, and how these two gradients are combined into a global temporal gradient for visuo-motor tasks. The general convention is that higher modules are executed faster than lower modules, and that the order of modules matters more than their absolute location. The left trapezium shows that the processing of location is achieved faster than the processing of intrinsic properties such as colour and shape. The right trapezium exhibits the temporal order of responses ranging from movement corrections to verbal judgement and comparison tasks. The dots on the central shaded arrow provide an estimate of the global duration for a specific visuo-motor task linking one attribute to one response. Bold lines correspond to data from Pisella et al. (1998, 2000). To allow for a loose comparison, thin lines correspond to results obtained by other studies quoted in the main text by using different timing criterion. 
two temporal gradients explains the striking speed observed for these specific location-correction tasks. The other extremity of this temporal gradient would be constituted by the combination of time-consuming processes in which ventral attributes are processed to produce a cognitive response such as a verbal description or a comparison (see Figure 4). A task within the central range of this gradient, such as processing a target colour or shape for making the decision about whether to point at or to avoid a given target in space would lead to intermediate durations. On the stimulus side, the duration of processing different attributes increases from the "Where" pole to the "What" pole. On the response side, the duration of motor output generation gradually increases from the "How" (pure and direct visuomotor response) pole to the "What" (identification response such as verbal report) pole. It is nevertheless interesting to note that location information is more frequently used for action responses, whereas conscious reports are more often associated with the integration of many object features including ventral properties. This further argues for the specific status of hand action with respect to perception and for its specialization in producing "sighted" fast responses to "blind" visual changes of a goal.

In addition to our timing analysis of sensori-motor tasks, several lines of arguments allow us to lighten the simple dichotic conception of vision. We will attempt to summarize here these arguments and suggest that there is more continuity between vision for perception and vision for action than is usually admitted.

First of all, the existence of a prolonged controversy between the "What vs. Where" conception and the "What vs. How" conception suggests that strictly dichotomous models may not be satisfactory. The anatomical division of visual pathways into two main streams has been initially considered as the substrate for "What" vs. "Where" processing (McIntosh et al., 1994; Ungerleider \& Haxby, 1994). Neuropsychological arguments from the double dissociation between optic ataxia and visual agnosia later raised the possibility that this anatomical division may instead relate to a distinction between "What" and "How" processing (Goodale \& Milner, 1992, Jeannerod \& Rossetti, 1993; Milner \& Goodale, 1995). It is also interesting to note that other neuropsychological evidence made the case for a double dissociation between knowing "What" and knowing "Where" (Wilson, Clare, Young, \& Hodges, 1997). These two conceptions have usually been regarded as mutually exclusive, the "How" being considered as a component of the "Where" processing by the tenants of the "What" vs. "Where" version, and the "Where" being recognized as useful mostly for knowing "How" to direct an action by the proponents of the "What" vs. "How" model. Although the aim of the present review is not to enter this debate, it may be mentioned here that knowing Where can be useful for purposes other than acting (e.g., verbal description of relative positions), and that knowing How to direct an action implies that not only space-based but also object-based visual information is processed (e.g., texture shape). Therefore, 
there should be space for processing both Where and How in the visual brain. Evidence for such a dissociation between Where and How has already been provided in the somatosensory system (Rossetti, Rode, \& Boisson, 1995). It will thus be important to seek further dissociations between Where and How in the different sensory domains, in order to confirm that "what" and "where" are not just two sides of one single function.

An informative PET study investigated the brain areas involved in performing motor and perceptual responses to visual objects (Faillenot, Toni, Decety, Gregoire, \& Jeannerod, 1997). The results showed that among activated cortical regions, some were specific to one task, but one was common to both. As expected from the What/How dissociation, different regions in the dorsal and ventral pathways were specifically activated during motor and perceptual responses, respectively. However, an additional region, located at the anterior part of the intra-parietal sulcus, was activated by both tasks. This region may correspond to the visual processing of an object's orientation or size, irrespective of whether the mode of response was motor or perceptual. Taken together, these specific and non-specific activations suggest not only that the same stimulus processing system can be involved in different tasks, but also that a module responsible for processing "Where" attributes in a large sense (object spatial characteristics) is distinct from a module responsible for the production of "How" responses.

Second, a continuum also seems to exist within the visuo-motor system itself, between parietal areas responsible for space-based action and those responsible for object-based action. Reversible inactivation in the monkey (Gallese, Murata, Kaseda, Niki, \& Sakata, 1994) and lesion studies in humans (Binkofski et al., 1998) indicate that the most rostral part of the intraparietal cortex is primarily involved in simple actions such as pointing, whereas the most ventral part is involved in grasping. This anatomical segregation may represent a first step of a more global gradient between two extremes, the prototype of which would be visual pointing (simple space-based action) and object recognition (object-based cognitive processing).

Third, the comparison of several psychophysical experiments, which individually support the dichotomy between vision for action and vision for perception, can lead to a different conclusion. On the one hand, and as described in the preceding sections, it has been shown that action and verbal reports can be fully dissociated in patients as well as in normal subjects, on spatial bases (Goodale, Milner, Jakobson, \& Carey, 1991; Goodale \& Milner, 1992; Jeannerod \& Rossetti, 1993; Milner \& Goodale, 1995), as well, as on temporal bases (see Bridgeman, 1991, 2000; Pisella \& Rossetti, 2000; Rossetti, 1998, 2000). Interestingly, in patients with damage to the ventral system, motor matching responses (such as indicating an object size or orientation with the hand) provide results as poor as results of purely perceptual tasks (e.g., Goodale, Jakobson, \& Keillor, 1994; Milner, Harvey, \& Pritchard, 1998; 
Perenin \& Rossetti, 1996). It is interesting to note that this dissociation also applies to objects oriented in the depth plane. Dijkerman, Milner, and Carey (1996; Dijkerman \& Milner, 1998) have compared reach-to-grasp and matching responses to objects oriented in depth. They found that the performance obtained for the two tasks could be dissociated in a patient with visual form agnosia as well as in normals, the orientation of the hand during prehension response being more linearly related to the actual object orientation than during the matching task.

On the other hand, further arguments for a dissociation between perception and action have been obtained by slope assessment experiments in normal human subjects. Bhalla and Proffitt (2000) have shown that a "motor" responses to 3D geographic slants (another example of orientation in the depth plane) are more veridical than a perceptual responses. In addition, delaying the responses alters this dissociation, such that delayed motor responses tend to follow the perceptual bias. The similarities in the effect of response delay between this study and the experiments previously referred to have led the latter authors to interpret their finding in the framework of the What vs. How version of the dorsal-ventral dissociation.

Comparison of these two series of study yields a different interpretation. Interestingly, the motor response used by Proffitt and collaborators is a haptic matching of the stimulus slope, which would be considered as a perceptual response on the basis of experiments using goal-directed action. Thus the same type of dissociation can be observed on one side between a goal-directed motor response and a manual matching response (linked to perception) and on the other side between a manual matching response (linked to action) and a perceptual verbal report. Taken together these behavioural studies suggest that manual matching responses may lie somewhere between the action and the perception sides of visually driven responses, thus introducing an intermediate term to the dichotomy that is usually proposed. In addition, brain imaging studies have shown that spatial location matching activates the superior parietal cortex (e.g., McIntosh et al., 1994), suggesting that the neuroanatomical substrate for this task is closer to the dorsal pathway than to the ventral pathway. Both behavioural and imaging thus results point to a possible intermediate level of visual processing that would fit in between the vision for action and the vision for perception systems. Other responses investigated may fall in the same category of intermediate task. For example, pantomiming a grasping action seems to give rise to results that are just between verbal report and the actual visuo-motor coordination at work during grasping, that is a less accurate but obvious grip scaling (see Goodale et al., 1994, Fig. 6B).

Fourth, as the dichotomy of the visual system has been best argued from the double dissociation between optic ataxia and visual agnosia (see Goodale \& Milner, 1992; Jeannerod \& Rossetti, 1993; Milner \& Goodale, 1995), meaningful information may be obtained from neuropsychological patients with lesions 
affecting intermediate cortical areas. One important feature of the deficit shown by optic ataxic patients is that their reaching difficulties are primarily exhibited in peripheral vision (e.g., Milner, Paulignan, Dijkerman, Michel, \& Jeannerod, 1999; Perenin \& Vighetto, 1988), suggesting that cortical areas outside the lesioned parietal areas are crucial for the visual guidance of movement near the centre of the visual field. It is, therefore, interesting to consider the effect of lesions lying in the brain area just between the focus of lesions producing optic ataxia and that of lesions producing visual agnosia: the temporo-parietal junction. The best documented deficit following such lesions in human is hemispatial neglect. On the one hand, a few experiments have attempted to contrast vision for action from vision for perception in neglect patients. They have suggested that the deficits observed in spatial parameters appear predominantly (Robertson, Nico, \& Hood, 1997) or even specifically (Perenin, 1997; Milner et al., 1998) on the perceptual side. This is coherent with the idea that neglect is primarily a deficit of awareness of visual processing in one half of space (Driver \& Mattingley, 1998). On the other hand, several temporal parameters have been used to show that visual neglect also involves some deficit on the action side (e.g., Mattingley, Phillips, \& Bradshaw, 1994; Mattingley, Husain, Rorden, Kennard, \& Driver, 1998). In accordance with our gradient hypothesis, it seems that brain lesions responsible for hemi spatial neglect, lying just between the dorsal and the ventral pathways, may produce intermediate disorders involving both some motor impairment and some cognitive impairment.

To conclude our speculations on the possible functional relationship between the two main visual systems, we should further consider the anatomical network involved. The dissociation between vision for action and vision for perception has been linked to the dorsal/ventral segregation of the cortical network of visual processing (Plate 1A: in order to see colour Plates 1A and 1B, please click here). However, as shown on Plate 1B, there are several cortical levels at which the dorsal and the ventral streams may interact. It is most interesting to note that there seems to be neither pure dorsal nor pure ventral projection onto the primary motor cortex (M1). Rather, these inputs result from projections from direct on indirect interconnected networks between the dorsal and the ventral stream. These anatomical connections may provide the substrate for visual processing intermediate between vision for perception and vision for action, and their existence is compatible with the continuum of visual functions proposed in this paper. Anatomical interactions located at the sensory, posterior level and involving direct and indirect connections have been given some attention in the past (e.g., Morel \& Bullier, 1990; Rossetti, 1998). In contrast, those at the motor, anterior level through converging projections to the frontal lobe have been largely ignored (with the notable exception of Schwartz, 1994) and should be emphasized in future studies. Indeed, the evidence reviewed here suggests that the respective contribution of the dorsal and the ventral streams to the processing of a given visual input may vary 
depending upon the behavioural tasks. For example, Plate 1B shows that, whereas there is a strong dominance of dorsal projections onto the dorsal premotor cortex (PMd), mainly involved in the control of arm-reaching movements, the ventral premotor cortex (PMv), which is mainly involved in the control of hand-grasping movements, receives direct projection from the ventral prefrontal cortex (PFv, which is a target area of the ventral stream). These relative dorsal/ventral contributions would provide a structural basis for the temporal gradient which can be observed between several visual functions as depicted in Figure 4.

Beyond the issue of strict anatomical localisation, the integrative problem is to know how extensively these systems interact together to produce an overt action (Pisella \& Rossetti, 2000; Rossetti \& Revonsuo, 2000). The study of the temporal dimension of visual processing, by showing how strongly specific behavioural functions are linked to given visual attribute processing, may shed new light on this debate. As argued here, the fast processing of location is compatible with the production of automatic motor corrections but this privileged link seems to exclude perceptual awareness on both functional and temporal bases. The timing of many possible tasks combining one stimulus and one response will be interesting to investigate in order to refine the sketch proposed on Figure 4 and further test our gradient hypothesis.

\section{REFERENCES}

Becker, W., \& Fuchs, A.F. (1969). Further properties of the human saccadic system: Eye movements and correction saccades with and without visual fixation points. Vision Research, 9, 1247-1258.

Bhalla, M., \& Proffitt, D. (2000). Geographical slant perception: Dissociation and coordination between explicit awareness and visually guided actions. In Y. Rossetti \& A. Revonsuo (Eds.), Beyond dissociation: Interaction between dissociated implicit and explicit processing (pp. 99-128). Amsterdam: Benjamins.

Binkofski, F., Dohle, C., Posse, S., Stephan, K.M., Hefter, H., Seitz, R.J., \& Freund, H.J. (1998). Human anterior intraparietal area subserves prehension: A combined lesion and functional MRI activation study. Neurology, 50, 1259.

Bridgeman, B. (1991). Complementary cognitive and motor image processing. In G. Obrecht \& L.W. Stark (Eds.), Presbyopia research: From molecular biology to visual adaptation (pp. 189-198). New York: Plenum Press.

Bridgeman, B. (2000). Complementary roles of cognitive and sensorimotor modes of visual processing. In Y. Rossetti \& A. Revonsuo (Eds.), Beyond dissociation: Interaction between dissociated implicit and explicit processing (pp. 17-40). Amsterdam: Benjamins.

Bridgeman, B., Hendry, D., \& Stark, L. (1975). Failure to detect displacement of the visual word during saccadic eye movements. Vision Research, 15, 719-722.

Bridgeman, B., Lewis, S., Heit, F., \& Nagle, M. (1979). Relation between cognitive and motor-oriented systems of visual perception. Journal of Experimental Psychology: Human Attention and Performance, 5, 692-700. 
Bridgeman, B., Peery, S., \& Anand, S. (1997). Interaction of cognitive and sensori-motor maps of visual space. Percept Psychophys, 59(3), 456-469.

Bridgeman, B., Van der Heijden, A.H.C., \& Velichkovsky, B. (1994). A theory of visual stability across saccadic eye movements. Behavioral and Brain Sciences, 17, 247-292.

Campbell, F.G., \& Wurtz, R.H. (1978). Saccadic omission: Why we do not see a grey-out during a saccadic eye-movement. Vision Research, 18, 1297-1303.

Castiello, U., \& Jeannerod, M. (1991). Measuring time to awareness. Neuroreport, 2, 797-800.

Castiello, U., Paulignan, Y., \& Jeannerod, M. (1991). Temporal dissociation of motor responses and subjective awareness: A study in normal subjects. Brain, 114, 2639-2655.

Desmurget, M., Pélisson, D., Rossetti, Y., \& Prablanc, C. (1998). From eye to hand: Planning goal directed movements. Neuroscience and Biobehavioural Reviews, 22, 761-788.

Desmurget, M., Prablanc, C., Arzi, M., Rossetti, Y., Paulignan, Y., \& Urquizar, C. (1996). Integrated control of hand transport and orientation during prehension movements. Experimental Brain Research, 110(2), 265-278.

Dijkerman, H.C., \& Milner, A.D. (1998). The perception and prehension of objects oriented in the depth plane: II. Dissociated orientation functions in normal subjects. Experimental Brain Research, 118, 408-414.

Dijkerman, H.C., Milner, A.D., \& Carey, D.P. (1996). The perception and prehension of objects oriented in the depth plane: I. Effect of visual form agnosia. Experimental Brain Research, $112,408-414$.

Dodge, R., (1900). Visual perception during eye movement. Psychological Review, 7, 454-465.

Driver, J., \& Mattingley, J.B. (1998). Parietal neglect and visual awareness. Nature Neuroscience, 1(1), 17-22.

Faillenot, I., Toni, I., Decety, J., Gregoire, M.C., \& Jeannerod, M. (1997). Visual pathways for object-oriented action and object recognition: Functional anatomy with PET. Cerebral Cortex, 7(1), 77-85.

Gallese, V., Murata, A., Kaseda, M., Niki, N., \& Sakata, H. (1994). Deficit of hand preshaping after muscimol injection in monkey parietal cortex. Neuroreport, 5(12), 1525-1529.

Goodale, M.A., \& Haffenden, A. (1998). Frames of reference for perception and action in the human visual system. Neuroscience and Biobehavioural Reviews, 22(2), 161-172.

Goodale, M.A., Jakobson, L.S., \& Keillor, J.M. (1994). Differences in the visual control of pantomimed and natural grasping movements. Neuropsychologia, 32, 1159-1178.

Goodale, M.A., \& Milner, A.D. (1992). Separate visual pathways for perception and action. Trends in Neurosciences, 15, 20-25.

Goodale, M.A., Milner, A.D., Jakobson, L.S., \& Carey, D.P. (1991). A neurological dissociation between perceiving objects and grasping them. Nature, 349, 154-156.

Goodale, M.A., Pélisson, D., \& PrablancE, C. (1986). Large adjustments in visually guided reaching do not depend on vision of the hand or perception of target displacement. Nature, 320(6064), 748-750.

Henson, D.B. (1978). Corrective saccades: Effects of altering visual feedback. Vision Research, 18, 63-67.

Jeannerod, M., \& Rossetti, Y. (1993). Visuo-motor coordination as a dissociable function: Experimental and clinical evidence. In C. Kennard (Ed.), Visual perceptual defects (Ballière's Clinical Neurology, International Practise and Research) (pp. 439-460). London: Ballière Tindall/ Saunders.

Komilis, E., Pélisson, D., \& Prablanc, C. (1993). Error processing in pointing at randomly feedback-induced double-step stimuli. Journal of Motor Behavior, 25(4), 299-308.

Mattingley, J.B., Husain, M., Rorden, C., Kennard, C., \& Driver, J. (1998). Motor role of human inferior parietal lobe revealed in unilateral neglect patients. Nature, 392, 179-182.

Mattingley, J.B., Phillips, J.G., \& Bradshaw, J.L. (1994). Impairments of movement execution in unilateral neglect: A kinematic analysis of bradykinesia. Neuropsychologia, 32(9), 11111134 . 
McIntosh, A.R., Grady, C.L., Ungerleider, L.G., Haxby, J.V., Rapoport, S.I., \& Horwitz, B. (1994). Network analysis of cortical visual pathways mapped with PET. Journal of Neuroscience, 14(2), 655-666.

Milner, A.D., \& Goodale, M.A. (1993). Visual pathways to perception and action. In T.P. Hicks, S. Molotchnikoff, \& T. Ono (Eds.), Progress in brain research (pp. 317-337).

Milner, A.D., \& Goodale, M.A. (1995). The visual brain in action (Oxford Psychology Series 27). Oxford, UK: Oxford University Press.

Milner, A.D., Harvey, M., \& Pritchard, C.L. (1998). Visual size processing in spatial neglect. Experimental Brain Research, 123, 192-200.

Milner, A.D., Paulignan, Y., Dijkerman, H.C., Michel, F., \& Jeannerod, M. (1999). A paradoxical improvement of misreaching in optic ataxia: New evidence for two separate neural systems for visual localization. Proceedings of the Royal Society (London), B, 266, 2225-2229.

Morel, A., \& Bullier, J. (1990). Anatomical segregation of two cortical visual pathways in the macaque monkey. Visual Neuroscience, 4, 555-578.

Nowak, L., \& Bullier, J. (1997). The timing of information transfer in the visual system. [In Cerebral cortex: Vol. 12, (Eds. J. Kaas, K. Rochland, \& A. Peters)] Extrastriate cortex in primates (pp. 205-241). New York: Plenum.

Paulignan, Y., \& Jeannerod, M. (1991). Selective perturbation of visual input during prehension movements: 2. The effect of changing object size. Experimental Brain Research, 87, 407-420.

Paulignan, Y., MacKenzie, C.L., Marteniuk, R.G., \& Jeannerod, M. (1991). Selective perturbation of visual input during prehension movements: 1 . The effect of changing object position. Experimental Brain Research, 83, 502-512.

Pélison, D., Prablanc, C., Goodale, M.A., \& Jeannerod, M. (1986). Visual control of reaching movements without vision of the limb. II. evidence of fast unconscious processes correcting the trajectory of the hand to the final position of a double-step stimulus. Experimental Brain Research, 62, 303-311.

Perenin, M.-T., (1997). Optic ataxia and unilateral neglect: Clinical evidence for dissociable spatial functions in posterior parietal cortex. In P. Their \& H.O. Karnath (Eds.), Parietal lobe contribution to orientation in 3D space (pp. 289-308). Heidelberg, Germany: Springer Verlag.

Perenin, M.-T., \& Rossetti, Y. (1996). Grasping without form discrimination in an hemianopic field. Neuroreport, 7(3), 793-797.

Perenin, M.-T., \& Vighetto, A. (1988). Optic ataxia: A specific disruption in visuomotor mechanisms. Brain, 11, 643-674.

Pisella, L., Arzi, M., \& Rossetti, Y. (1998). The timing of colour and location processing in the motor context. Experimental Brain Research, 121(3), 270-276.

Pisella, L., Gréa, H., Tilikete, C., Vighetto, A., Desmurget, M., Rode, G., Boisson, D., \& Rossetti, Y. (in press). An automatic pilot for the hand in the human posterior parietal cortex. Nature Neuroscience.

Pisella, L., Kosawa, R., Susani, K., \& Rossetti, Y. (2000). The timing of form processing in the motor context. Unpublished manuscript.

Pisella, L., \& Rossetti, Y. (1997). Stimulus location is processed faster than stimulus color. Perception, 26(Suppl.), 100.

Pisella, L., \& Rossetti, Y. (2000). Interaction between conscious identification and non-conscious sensori-motor processing: Temporal constraints. In. Y. Rossetti \& A. Revonuso (Eds.), Beyond dissociation: Interaction between dissociated implicit and explicit processing (pp. 129-152). Amsterdam: Benjamins.

Prablanc, C., \& Jeannerod, M. (1975). Corrective saccades: Dependence on retinal reafferent signals. Vision Research, 15, 465-469.

Prablanc, C., \& Martin, O. (1992). Automatic control during hand reaching at undetected twodimensional target displacements. Journal of Neurophysiology, 67(2), 465-469.

Robertson, I.H., Nico, D., \& Hood, B.M. (1997). Believing what you feel: Using proprioceptive feedback to reduce unilateral neglect. Neuropsychology, 11(1), 53-58. 
Rossetti, Y. (1998). Short-lived motor representation of space in brain-damaged and healthy subjects. Consciousness and Cognition, 7(3), 520-558.

Rossetti, Y. (1999). In search of immaculate perception: Evidence from motor perception of space. In S. Hameroff, A. Kaszniak, \& D. Chalmers (Eds.), Towards a science of consciousness (pp. 141-148). Cambridge, MA: MIT Press.

Rossetti, Y. (2000). Implicit perception in action: Short-lived motor representations of space. In P.G. Grossenbacher (Ed.), Finding consciousness in the brain (pp. 131-179). Amsterdam: Benjamins.

Rossetti, Y., Gaunet, F., \& Thinus-Blanc, C. (1996). Early visual experience affects memorization and spatial representation of proprioceptive targets. Neuroreport, 7, 1219-1223.

Rossetti, Y., \& Pisella, L. (1998). Temporal asynchrony between sensory and between motor components of a visuo-motor response: A time-grounded dissociation between implicit and explicit processing? Journal of Consciousness Studies. (Consciousness Research Abstract, I, 10. 108).

Rossetti, Y., \& Procyk, E. (1997). What memory is for action: The gap between percepts and concepts. Behavioral and Brain Sciences, 20(1), 34-36.

Rossetti, Y., \& Régnier, C. (1995). Representations in action: Pointing to a target with various representations. In B.G. Bardy, R.J. Bootsma, \& Y. Guiard (Eds.), Studies in perception and action III (pp. 233-236). Mahwah, NJ: Lawrence Erlbaum Associates Inc.

Rossetti, Y., \& Revonsuo, A. (2000). Beyond dissociations: Recomposing the mind-brain after all? In Y. Rossetti \& A. Revonsuo (Eds.), Beyond dissociation: Interaction between dissociated implicit and explicit processing (pp. 1-16). Amsterdam: Benjamins.

Rossetti, Y., Rode, G., \& Boisson, D. (1995). Implicit processing of somesthetic information: A dissociation between where and how? Neuroreport, 6(3), 506-510.

Schall, J.D., Morel, A., King, D.J., \& Bullier, J. (1995). Topography of visual cortex connections with frontal eye field in macaque: Convergence and segregation of processing streams. Journal of Neuroscience, 15, 4464-4487.

Schmolensky, M.T., Wang, Y., Hanes, D.P., Thompson, K.G., Leutgeb, S., Schall, J.D., \& Leventhal, A.G. (1998). Signal timing across the macaque visual system. Journal of Neurophysiology, 79, 3272-3278.

Schwartz, A.B. (1994). Distributed motor processing in cerebral cortex. Current Opinion in Neurobiology, 4, 840-846.

Simons, D.J. (1996). In sight, out of mind: When object representations fall. Psychological Science, 7(5), 301-305.

Tanaka, Y., \& Shimojo, S. (1995). Location vs feature: Reaction time reveals dissociation between two visual functions. Vision Research, 36, 2125-2140.

Tanné, J., Boussaoud, D., Boyer-Zeller, N., \& Rouiller, E. (1995). Direct visual pathways for reaching movements in the macaque monkey. Neuroreport, 7, 267-272.

Ungerleider, L.G., \& Haxby, J.V. (1994). "What" and "where" in the human brain. Current Opinion in Neurobiology, 4(2), 157-165.

Ungerleider, L.G., \& Mishkin, M. (1982). Two cortical visual systems. In D.J. Ingle, M.A. Goodale, \& R.J.W. Mansfield (Eds.), Analysis of visual behaviour. Cambridge, MA: MIT Press.

Van Hoesen, G.W. (1982). The parahippocampal gyrus: New observations regarding its cortical connections in the monkey. Trends in Neurosciences, October, 345-350.

Wilson, B.A., Clare, L., Young, A.W., \& Hodges, J.R. (1997). Knowing where and knowing what: A double dissociation. Cortex, 33(3), 529-541. 
Plates 1A and 1B (Please click here). Neuroanatomical basis for dissociated perception and action systems.

A: Schematic drawing of the two main neural pathways in the visual system. From the main visual input to area 17, two segregated streams of processing have been described projecting respectively to the posterior parietal cortex (dorsal pathway) and to the inferotemporal cortex (ventral pathway). Whereas the ventral pathway is specialized in processing colour and form, and is assumed to play a key role in object identification ("What"), the dorsal pathway is known to be primarily involved in the computation of places ("Where") and in the sensori-motor processing of the object metrics ("How"). A temporal dissociation between these two streams is argued in the text.

B: Cortical neuronal networks allowing visual inputs to be transformed into motor output. Although the dorsal and the ventral streams can be individuated from this network, this illustration emphasizes the reductionism presented on Plate $1 \mathrm{~A}$, and displays possible substrate for interactions between these two main pathways driving information from VI to MI. The dorsal and the ventral streams are depicted here in green and red respectively, as well as their efferences. Blue arrows arise from areas receiving convergent dorsal and ventral inputs, either directly or indirectly. Further projections from areas receiving these mixed convergent inputs have also been represented in blue. Even though the posterior parietal cortex and the inferior temporal cortex receive a single direct projection from each other, they were not considered as mixed recipient areas. By contrast, areas in the frontal lobe receive parallel dorsal, ventral, and mixed projections. Interestingly, M1 receives only pure dorsal projections and mixed projections, but no pure ventral projections. Since recent reviews have focused on the role of sub-cortical areas in visuo-motor processing, such as the projections from the superior colliculus via the pulvinar to the posterior parietal lobe and the inferior temporal lobe (e.g., Milner \& Goodale, 1995; Rossetti, 1998), this figure primarily depicts cortical networks. AIP: anterior intraparietal area; BS: brainstem; Cing: Cingulate motor areas; d: dorsal; FEF: frontal eye field, FST: floor of the superior temporal sulcus; Hipp: Hippocampus; LIP: lateral intraparietal area; M1: primary motor cortex; MIP: mesial intraparietal area; MST: medial superior temporal area; MT: medio-temporal area; PR: prefrontal cortex; PM: premotor cortex; SC: superior colliculus; SEF: supplementary eye field, SMA: supplementary motor area; STS: superior temporal sulcus; STP: superior temporal polysensory area; TE: temporal area; TEO: temporo-occipital area; v: ventral; V1: primary visual cortex; VIP: ventral intraparietal area. (Derived from Morel \& Bullier, 1990; Schwarz, 1994; Schall, Morel, King, \& Bullier, 1995; Tanné, Boussaoud, Boyer-Zeller, \& Rouiller, 1995; Van Hoesen, 1982.) 
(A)

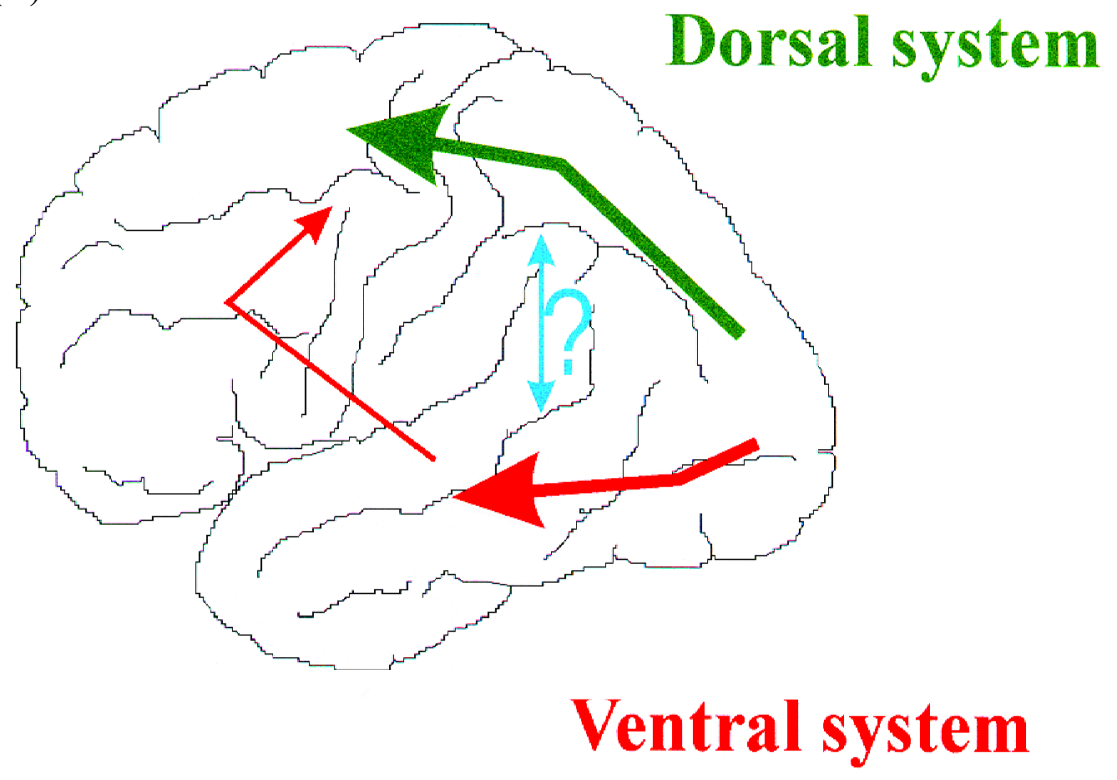

(B)

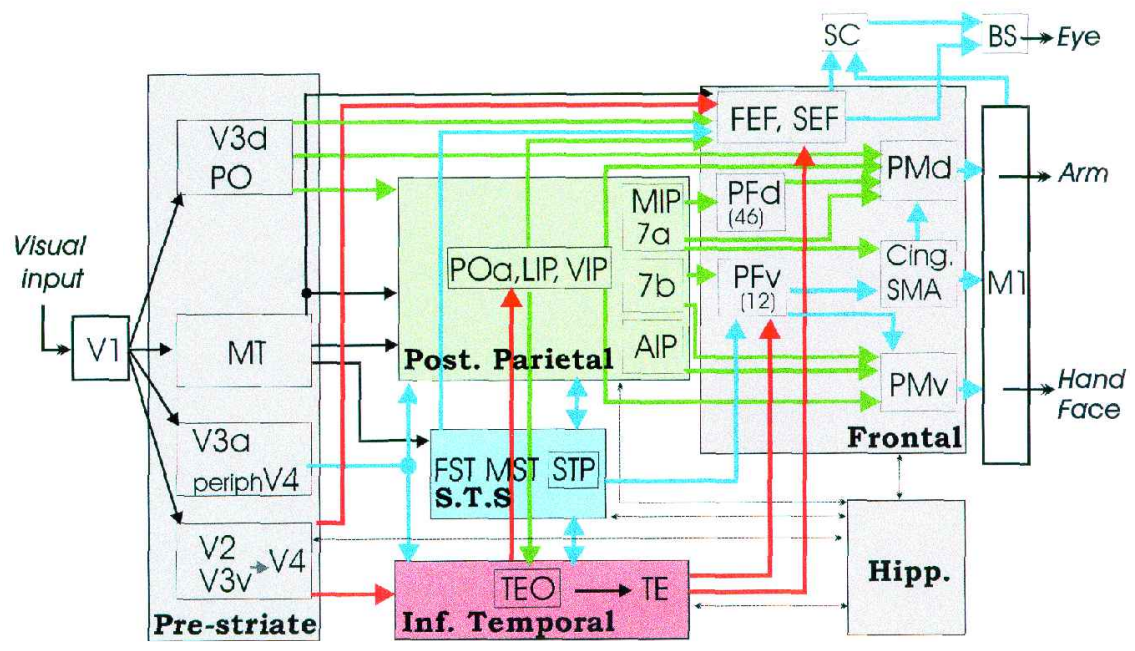

